\title{
Baroclinic Eddies and the Extent of the Hadley Circulation: An Idealized GCM Study
}

\author{
XAVIER J. LEVINE \\ Yale University, New Haven, Connecticut \\ TAPIO SCHNEIDER \\ ETH Zürich, Zurich, Switzerland, and California Institute of Technology, Pasadena, California
}

(Manuscript received 27 May 2014, in final form 31 January 2015)

\begin{abstract}
The Hadley circulation has widened over the past 30 years. This widening has been qualitatively reproduced in general circulation model (GCM) simulations of a warming climate. Comprehensive GCM studies suggest this widening may be caused by a poleward shift in baroclinic eddy activity. Yet the limited amplitude of the climate change signals analyzed so far precludes a quantitative comparison with theories.

This study uses two idealized GCMs, one with and one without an active hydrologic cycle, to investigate changes in the extent of the Hadley circulation over a wide range of climates. The climates span global-mean temperatures from 243 to $385 \mathrm{~K}$ and equator-to-pole temperature contrasts from 12 to $100 \mathrm{~K}$. Baroclinic eddies control the extent of the Hadley circulation across most of these climates. A supercriticality criterion that quantifies the depth of baroclinic eddies relative to that of the troposphere turns out to be a good indicator of where baroclinic eddies become deep enough to terminate the Hadley circulation. The supercriticality depends on meridional temperature gradients and an effective stability that accounts for the effect of convective heating on baroclinic eddies.

As the equator-to-pole temperature contrast weakens or the convective static stability increases, convective heating increasingly influences the thermal stratification of the troposphere and the supercriticality. Consistent with the supercriticality criterion, the Hadley circulation contracts as meridional temperature gradients increase, and it widens as the effective static stability increases. The former occurs during El Niño and may account for the observed Hadley circulation contraction then; the latter occurs during global warming.
\end{abstract}

\section{Introduction}

Observational studies suggest that the Hadley circulation in the tropics has widened over the past 30 years (Hu and Fu 2007; Seidel and Randel 2007; Seidel et al. 2008), although there is a wide spread of the magnitude of the widening among datasets (Adam et al. 2014). The widening has been qualitatively reproduced in general circulation model (GCM) simulations of global warming scenarios. GCM simulations agree that the terminus of the Hadley cells shifts poleward under global warming, but they disagree on the magnitude of this shift (e.g., Lu et al. 2007). The shift is accompanied by a poleward shift of the subtropical dry zones (e.g., Lu et al. 2007) and midlatitude storm tracks (e.g., Yin 2005; Barnes and

Corresponding author address: Xavier J. Levine, Yale University, 210 Whitney Ave., New Haven, CT 06511.

E-mail: xavier.levine@yale.edu
Polvani 2013; Simpson et al. 2014). At the same time, the Hadley circulation has been observed to contract during El Niño and to expand during La Niña (e.g., Seager et al. 2003; Lu et al. 2008; Nguyen et al. 2013; Adam et al. 2014).

There is no convincing account of what causes the widening trend or ENSO variations of the Hadley circulation; a theory describing the width of the Hadley circulation on an "Earth like" planet is lacking (Schneider 2006; Schneider et al. 2010). The nowprevalent hypothesis is that the Hadley circulation terminates where baroclinic eddies in some sense start controlling the dynamics. Several arguments of how this may occur have been put forward. The simplest version of this argument is based on the linear stability criterion for the quasigeostrophic (QG) two-layer model and states that the Hadley circulation extends up to the latitude at which angular momentum-conserving axisymmetric flow becomes baroclinically unstable (Held 
2000). This argument, and its quantitative consequences, has been widely used in the recent literature to account for the poleward shift of the Hadley circulation terminus in comprehensive GCM simulations ( $\mathrm{Lu}$ et al. 2007; Frierson et al. 2007). Yet the resulting Hadley circulation extent compares poorly with idealized GCM simulations over a much wider range of climates than that sampled in the comprehensive GCM simulations (Walker and Schneider 2006; Korty and Schneider 2008). Moreover, its core assumptions-angular momentum-conserving flow in the upper branch of the Hadley circulation and the existence of a critical shear for baroclinic instabilityare violated in Earth's atmosphere (Schneider 2006; Zurita-Gotor and Lindzen 2007).

A modified version of this argument is based on a diffusive eddy mixing model and posits that the Hadley circulation extends to the latitude where baroclinic eddies become deep enough to reach the upper troposphere and where, as a consequence, the eddy flux divergence of angular momentum changes sign (Schneider and Walker 2006; Korty and Schneider 2008). Determining this latitude from a supercriticality criterion accounts broadly for Hadley circulation changes in simulations with a dry idealized GCM (Korty and Schneider 2008). But applying this criterion to an atmosphere with an active hydrologic cycle has remained challenging (Schneider and O'Gorman 2008). More recently, O'Gorman (2011) suggested a way to include moisture effects in the depth scaling of baroclinic eddies. This modified scaling was found to capture changes in the extent of the Hadley circulation in global warming simulations with an idealized GCM with an active hydrologic cycle (O'Gorman 2011). However, these recent findings have raised new questions: for example, whether the supercriticality in dry and moist GCM simulations can be represented by a uniform framework, despite the differences in which diabatic heating occurs. It is unclear to what extent convective heating in dry GCM simulations affects baroclinic eddies and whether an effective static stability similar to that of O'Gorman (2011) captures its effects. Furthermore, it is unclear whether accounting solely for vertical convection can capture the effects of diabatic heating on baroclinic eddies or whether other processes, such as slantwise convection, need to be represented explicitly as well (Korty and Schneider 2007).

Here, we describe a modified supercriticality criterion, which is very similar to that defined by O'Gorman (2011), and we test its relevance over a wide range of climates simulated with two idealized GCMs. We show that this criterion can discriminate between regions dominated by baroclinic eddy or convective activity and that it constrains changes in the Hadley circulation extent with climate when baroclinic wave activity is strong in the extratropics.

\section{Heuristic arguments}

The simplest model of the Hadley circulation is that of an angular momentum-conserving axisymmetric overturning circulation that is energetically closed (Schneider 1977; Held and Hou 1980). Held and Hou (1980) showed that such a Hadley circulation extends to a finite latitude, beyond which radiative-convective equilibrium prevails. In our simulations, the prediction of the Hadley cell extent obtained from this dynamical model compares poorly with the actual latitude of the Hadley circulation terminus (see appendix C). This is unsurprising because the tropical circulation is neither angular momentum conserving nor energetically closed (Walker and Schneider 2006; Schneider 2006; Trenberth and Stepaniak 2003).

Axisymmetric flows were not originally viewed as accounting for the behavior of Earth's Hadley circulation, but as providing a basic state for studies of baroclinic instability (e.g., Schneider 1977). The zonal wind speeds consistent with an angular momentumconserving mean flow would be large enough in the subtropics to be linearly unstable to baroclinic instability (e.g., Phillips 1954). This led to the notion that the axisymmetric Hadley circulation may extend up to the latitude where baroclinic instability "sets in" (Held 2000). The analytical relation for an angular momentum-conserving axisymmetric Hadley circulation terminated by linear baroclinic instability has been used in the recent literature to explain the poleward shift of the Hadley circulation terminus in comprehensive GCM simulations (e.g., Lu et al. 2007; Frierson et al. 2007; Kang and Lu 2012). But a quantitative comparison of the resulting Hadley circulation extent with that in our idealized GCM simulations shows significant discrepancies (see appendix C), consistent with previous findings (Walker and Schneider 2006; Korty and Schneider 2008). This is again not surprising, because core assumptions, such as the tropical upper troposphere being in an angular momentum-conserving regime, are usually violated in Earth's atmosphere (Walker and Schneider 2006; Schneider 2006).

More generally, the Hadley circulation terminus may be considered as the equatorward boundary of the region where baroclinic eddies become deep enough to reach the upper troposphere (Fig. 1). Where they reach the upper troposphere, wave activity no longer propagates upward but horizontally, implying angular momentum flux convergence into the latitude band where it is generated (Edmon et al. 1980; Held and Hoskins 1985; 


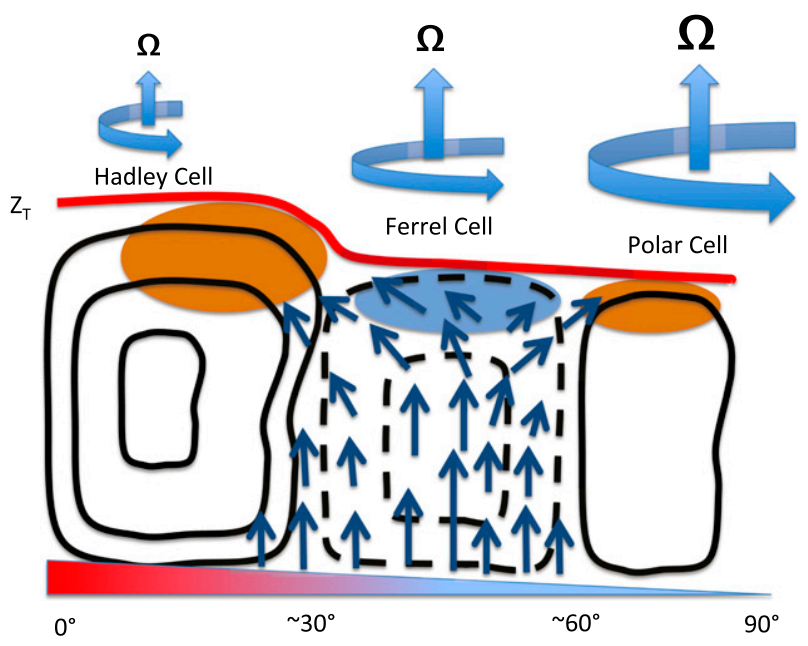

FIG. 1. Cartoon of the effect of macroturbulence on the extent of the Hadley circulation. When the thermal contrast becomes large enough, baroclinic waves set the height of the tropopause (red line). A change in the sign of the potential vorticity gradient between the surface layer (as shown by temperature decreasing poleward at the surface) and the interior of the troposphere (as shown by planetary vorticity increasing poleward) triggers baroclinic instability near the surface. Eddies then propagate upward and equatorward into the subtropical upper troposphere, where they dissipate (blue arrows). The terminus of the Hadley circulation corresponds to a boundary between regions of eddy dissipation (subtropics) and eddy production (middle latitudes). In the upper troposphere, this is characterized by a transition between a region with eddy momentum flux divergence (orange patches) and poleward mean meridional winds (Hadley cell; solid black contours) to a region of eddy momentum flux convergence (blue patch) and equatorward mean meridional winds (Ferrel cell; dashed black contours).

Vallis 2006; Ait-Chaalal and Schneider 2015). Because the Rossby number in the descending branch of the Hadley circulation and poleward of it is generally small (Walker and Schneider 2006; Levine and Schneider 2011), this implies equatorward mean meridional flow where baroclinic eddies are generated and reach the upper troposphere. In this view, the terminus of the Hadley circulation defines the equatorward margin of wave activity generation and upward propagation into the upper troposphere (Korty and Schneider 2008).

\section{a. Supercriticality in dry atmospheres}

Building on Held (1978), Schneider and Walker (2006) derived a nondimensional criterion, called supercriticality:

$$
S_{c}=\frac{\Delta_{h} \theta}{\Delta_{v} \theta} \approx \frac{\bar{p}_{o}-\bar{p}_{e}}{\bar{p}_{o}-\bar{p}_{t}}
$$

which quantifies the ratio of the depth scale of baroclinic entropy fluxes to the height of the tropopause in dry atmospheres. Here, the overbar defines a temporal and zonal mean; $\bar{p}_{o}$ is the mean surface pressure, $\bar{p}_{t}$ is the mean tropopause pressure (determined as described in appendix $\mathrm{B}$ ), and $\bar{p}_{e}$ is the mean pressure to which substantial eddy entropy fluxes extend; $\Delta_{h} \theta$ is a meridional potential temperature contrast, defined with the near-surface meridional gradient $\left(\partial_{y} \bar{\theta}_{s}\right)$ of potential temperature as

$$
\Delta_{h} \theta=-\frac{f}{\beta} \partial_{y} \bar{\theta}_{s},
$$

and $\Delta_{v} \theta$ is a near-surface static stability measure, defined with the near-surface static stability $\left(-\partial_{p} \bar{\theta}_{s}\right)$ as

$$
\Delta_{v} \theta=-2 \partial_{p} \bar{\theta}_{s}\left(\bar{p}_{o}-\bar{p}_{t}\right) .
$$

The near-surface average $(\cdot)_{s}$ is taken to be the horizontal mean averaged between the $800-$ and $700-\mathrm{hPa}$ pressure levels, that is, just above the planetary boundary layer.

In regions where baroclinic wave activity strongly affects the mean flow and thus sets the depth of the troposphere, Schneider and Walker (2006) showed that for Earth-like climates, $S_{c} \simeq 1$. In regions where baroclinic eddies are shallower than the depth of the troposphere, $S_{c}<1$. The latter occurs where radiative-convective processes set the tropopause height, for example, in the tropics. To obtain relation (1) for the depth of baroclinic eddy entropy fluxes, Schneider and Walker (2006), following Held (1978), assumed that potential vorticity (PV) along dry isentropes is mixed in the troposphere, that potential temperature is mixed along the surface, and that the turbulent diffusivity has no essential vertical structure (i.e., that the mixing is essentially barotropic over the depth over which baroclinic eddies extend). The latter is expected in the limit of strong turbulence and is an approximation when turbulence is weak (cf. Held 1978). Relation (1) is strictly valid only when applied on length scales greater than that of the eddies, which are often of planetary scale (see appendix A for a definition of this mixing region).

Using an idealized dry GCM like the one that we use here, Schneider and Walker (2006) found that $S_{c}$, when averaged over large-scale regions of strong baroclinic eddy activity, assumed $O(1)$ values over a wide range of climates. Values of $S_{c} \sim 1$ were attained when the tropospheric thermal stratification was controlled by baroclinic eddies. Values of $S_{c}<1$ were attained when convection stabilized the tropospheric thermal stratification more than baroclinic eddies could, that is, when baroclinic eddies were relatively weak.

Supercritical climates with $S_{c} \gg 1$ are possible if one or more assumptions leading to the $S_{c} \lesssim 1$ constraint are 
not satisfied. For example, supercritical climates can occur when the stratification and meridional potential temperature gradients are much weaker than in Earth's atmosphere so that the near-surface static stability approaches zero (Schneider and Walker 2006; Jansen and Ferrari 2012). Or if the radiative time scales become comparable or shorter than dynamical time scales, eddies become inefficient at mixing PV and near-surface potential temperatures. In that case, climates with $S_{c} \geq 1$ can arise if the radiative-convective equilibrium state to which the climate strongly relaxes has $S_{c}>1$ (e.g., Chai and Vallis 2014). Mildly supercritical climates can also occur when turbulence is weak and the turbulent diffusivity inherits a nonbarotropic structure from the mean flow. However, our focus is on Earth-like climates, for which $S_{c} \lesssim 1$ appears to hold well.

The supercriticality (1) with $S_{c} \simeq 1$ is formally similar to the necessary condition for baroclinic instability in the QG two-layer model (Phillips 1954). Stone (1978) and Stone and Nemet (1996) used this criterion to suggest that Earth's extratropical troposphere is close to neutral with respect to linear baroclinic instability. Others have used the linear-stability interpretation of $S_{c} \simeq 1$ to explain poleward shifts in the terminus of the Hadley circulation with global warming, combining the criterion either with an assumption of axisymmetric flow in the tropics (e.g., Held 2000; Vecchi and Soden 2007; Frierson et al. 2007) or with the actual zonal-mean flow (e.g., Tandon et al. 2013). Despite the formal similarity, however, the interpretations of the supercriticality (1) and of the necessary condition for baroclinic instability in the QG two-layer model differ. The supercriticality (1) does not assume or imply a state of marginal stability, which is known not to be attained in the atmosphere (Simmons and Hoskins 1980; Barry et al. 2000; ZuritaGotor and Lindzen 2007; Merlis and Schneider 2009). Rather, the constraint $S_{c} \lesssim 1$ emerges from considering the limit of strong turbulence and how it would affect the atmospheric thermal structure.

These results for dry atmospheres suggest that one may use $S_{c}$ to locate the terminus of the Hadley circulation in climates in which baroclinic eddies influence the thermal stratification (see Fig. 1). Korty and Schneider (2008) defined a local $S_{c}$ as the ratio of the local depth of the entropy fluxes at the latitude of the terminus over the depth of the extratropical troposphere. They found that the Hadley circulation in many climates simulated with a dry GCM extends to the latitude at which the local $S_{c}$ first exceeds a critical $O(1)$ value. (The value of $S_{c}$ generally increases with latitude because the geometric term $f / \beta=a \tan \phi$ increases rapidly with latitude $\phi$.) Here, we build on that work and extend the results to regimes in which convection and latent heat release are important in controlling the subtropical and extratropical thermal stratification.

\section{b. Effective static stability}

Schneider and Walker (2006) derived the supercriticality (1) neglecting moisture effects on baroclinic eddies. However, latent heat release in large-scale condensation and moist convection are known to affect the intensity and structure of baroclinic eddies (e.g., Emanuel et al. 1987; Gutowski et al. 1992; Lapeyre and Held 2004), as well as the tropospheric thermal stratification (Korty and Schneider 2007). Using an idealized moist GCM, Schneider and O'Gorman (2008) found a considerable effect of moisture on $S_{c}$ : as the climate warmed and moisture effects became more prevalent, the supercritality decreased to values $S_{c}<1$, in contrast to its near invariance found in the dry GCM simulations. Only in the coldest moist simulations were $O(1)$ values of supercriticality observed. Schneider and O'Gorman (2008) attributed this decrease with global warming to an increase in the static stability and tropopause height, associated with increasing surface temperature and large-scale condensation in moist baroclinic eddies. This points to the need for a modified static stability measure that accounts for latent heat release in baroclinic eddies. Such an effective static stability was proposed by O'Gorman (2011), whose ideas we briefly review here.

To devise a theory for an effective static stability that takes latent heat release in eddies into account, O'Gorman (2011) addressed the asymmetry that exists in moist atmospheres between updrafts, which favor moisture saturation and latent heat release, and downdrafts without condensation. This asymmetry affects the atmospheric dry entropy budget:

$$
\partial_{t} \theta+\cdots+\omega \partial_{p} \theta=\cdots+Q_{c} .
$$

Here, $Q_{c}$ refers to latent heat release associated with condensation of water, which dominates locally over other diabatic processes, such as radiative heating, when condensation occurs. Changes in the dry entropy of a saturated air parcel during condensation imply a vertical displacement (O'Gorman 2011); if the distance to saturation is much larger in the horizontal than in the vertical direction (as is typically the case in an Earth-like climate), then latent heat release becomes a function of local upward velocity and static stability:

$$
Q_{c}=\omega^{\uparrow} \partial_{p} \theta^{\uparrow} \mathcal{H}(-\omega) .
$$

Here, $\omega^{\uparrow}$ denotes an upward velocity in pressure coordinates, $\partial_{p} \theta^{\uparrow}$ is the static stability in updrafts, and 
$\mathcal{H}(-\omega)$ is the Heaviside function, which selectively removes downdrafts.

While O'Gorman (2011) considered $\partial_{p} \theta^{\uparrow}$ to depend on local thermodynamic conditions, in our study we take it to be in equilibrium with near-surface conditions in a column. That is, updrafts and condensation in the free troposphere are tied to the thermodynamic state of the boundary layer, with deep convection acting as communicator:

$$
\partial_{p} \theta^{\uparrow}=\left.\partial_{p} \theta\right|_{\theta_{o}^{*}}
$$

where $-\left.\partial_{p} \theta\right|_{\theta_{o}^{*}}$ is the static stability set by air parcels lifted pseudoadiabatically from the surface and conserving their near-surface moist entropy $\theta_{o}^{*}$ in the process. In the extratropics, deep baroclinic eddies set zones of deep ascent around cyclones or along frontal zones separating warm subtropical air and cold polar air masses: the regions of ascent are collocated with vigorous convective activity (Ralph et al. 2004; Knippertz and Wernli 2010).

While the net upward velocity $\omega^{\uparrow}$ cannot be constrained without a detailed description of the local energy budget, O'Gorman (2011) provided a geometric argument that relates the eddy upward velocity approximately to the total eddy velocity:

$$
\omega^{\uparrow \prime}=\lambda \omega^{\prime}+\varepsilon .
$$

Here, $\lambda$ is a rescaling factor and $\varepsilon$ is a residual. Relation (7) is satisfied identically when the eddy velocity structure is binary either up or down, in which case $\lambda$ defines the surface area covered by downdrafts. But relation (7) also is an adequate approximation for other (more realistic) eddy velocity structures [see O'Gorman (2011) for details]. Dry eddies are expected to show equipartition between updrafts and downdrafts (i.e., $\lambda=0.5$ ). In Earth's atmosphere, NCEP-2 reanalyses show that $\lambda$ converges to 1 in the deep tropics, where updrafts are confined to a small part of the domain, while in the extratropics its value is near 0.55 , consistent with near equipartition there (O'Gorman 2011). This value was found to be nearly invariant in the extratropics over a wide range of climates, which suggests that relation (7) is a useful representation of the eddy updraft velocity (O'Gorman 2011; Booth et al. 2015).

Using near-convective neutrality to compute the static stability in updrafts (6) and the linearized expression for eddy upward velocity (7), the eddy entropy budget may be expressed as

$$
\partial_{t} \theta^{\prime}=-\omega^{\prime} \overline{\partial_{p} \theta^{\mathrm{eff}}}
$$

where the effective static stability experienced by baroclinic eddies averaged over many life cycles is

$$
\overline{\partial_{p} \theta^{\text {eff }}}=\partial_{p} \bar{\theta}-\lambda \overline{\left.\partial_{p} \theta\right|_{\theta_{o}^{*}}} .
$$

This effective static stability now incorporates the latent heat release (5) and thus makes the associated diabatic heating, which is external in dry dynamics, internal to the dynamics. An effective static stability measure similar to the dry static stability measure (3) can then be defined as

$$
\Delta_{v}^{\mathrm{eff}} \theta=-2 \overline{\partial_{p} \theta_{s}^{\mathrm{eff}}}\left(\bar{p}_{o}-\bar{p}_{t}\right)
$$

In a moist atmosphere, the effective static stability measure (10) is always smaller than the dry static stability measure (3), consistent with the added buoyancy provided by condensation and latent heat release. In a dry atmosphere, the static stability $\left(-\partial_{p} \bar{\theta}\right)$ equals its effective form $\left(-\overline{\partial_{p} \theta^{\mathrm{eff}}}\right)$ because $-\overline{\left.\partial_{p} \theta\right|_{\theta_{o}^{*}}}=0$. Whenever the convective lapse rate is more stable than its dry adiabatic limit, a reduced effective static stability is felt by eddies regardless of any other moisture effect. This has important implications for how eddies equilibrate in our dry GCM, in which the convective lapse is prescribed to be stable and moist like, to achieve greater resemblance with Earth's climate.

There is no significant difference in the moist simulations between our estimates of the effective static stability measure (10) and that defined by O'Gorman (2011). Indeed, relation (10) is a specific case of that in O'Gorman (2011), because saturated equivalent entropy is conserved in saturated updrafts whether or not it is associated with vertical convection. But the scaling devised by O'Gorman (2011) cannot easily be tested in dry GCM simulations, because an equivalent potential temperature cannot easily be defined there. This contrasts with our scaling, which can be tested in dry GCM simulations using the prescribed convective lapse rate. The more restrictive interpretation of our effective static stability measure (10) allows for a wider applicability when compared to that defined in O'Gorman (2011). But both estimates are nearly identical when both versions of effective static stability are applicable, such as in moist GCM simulations.

With the effective static stability measure (10), a heuristic effective supercriticality $S_{c}^{\text {eff }}$ can be defined. It is identical to relation (1) except for the replacement of the dry static stability by the effective moist static stability. The effective supercriticality is systematically greater than its dry form. O'Gorman (2011) found the effective supercriticality to be invariant at the terminus of the Hadley circulation, assuming a value $S_{c}^{\text {eff }} \approx 0.6$, in moist GCM simulations under global warming; this value is significantly larger than the dry supercriticality $S_{c} \approx 0.4$ found in the dry GCM simulations of Korty and Schneider (2008), or in the moist GCM simulations of 
Schneider and O'Gorman (2008) in the limit of vanishing moisture content.

\section{Idealized GCMs}

We examine whether the effective supercriticality and the constraint $S_{c}^{\text {eff }} \simeq 1$ can account for the extratropical thermal stratification and the extent of the Hadley circulation in a wide range of simulations with idealized GCMs. We use a dry GCM to investigate the effect of convective mixing on the supercriticality independent of other moisture effects (e.g., large-scale condensation). And we use a moist GCM to investigate more broadly how moist convection and moisture effects in baroclinic eddies affect the extratropical thermal structure and Hadley circulation extent.

\section{a. Idealized dry GCM}

The idealized dry GCM is described in Schneider and Walker (2006). It simulates an atmosphere bounded by a spatially uniform and thermally insulating spherical surface. A spectral dynamical core solves for the large-scale motions in the atmosphere, with a resolution of T85 in all experiments. The vertical coordinate is discretized with 30 sigma levels. Momentum and dry entropy are diffused in a boundary layer of fixed height $(2.5 \mathrm{~km})$, with a turbulent Prandtl number of 1 (Smagorinsky et al. 1965). Frictional dissipation at the surface is parameterized by a bulk aerodynamic formula. Radiative heating is represented by Newtonian relaxation of potential temperatures toward a zonally symmetric radiative equilibrium profile, which is quadratic in the cosine of latitude and statically unstable to dry convection. The time scale for this Newtonian relaxation is zonally symmetric and varies with latitude and height from 7 days at the equator near the surface to 50 days in the interior atmosphere (Schneider 2004). Convection is parameterized by a quasi-equilibrium convection scheme. The convective lapse rate is varied by rescaling the dry adiabatic lapse rate by a rescaling factor $\gamma \leq 1$. Using a reduced convective lapse rate mimics changes in the stability of a convective atmosphere due to an increase in the nearsurface moisture content: decreasing $\gamma$ from its dry adiabatic value of 1 is similar to increasing the moisture content just above surface, which usually happens in a moist atmosphere as a response to warmer surface temperatures.

We simulated a wide range of climates by varying the equator-to-pole temperature contrast in radiative equilibrium from 15 to $300 \mathrm{~K}$ and the convective lapse rate rescaling factor from 0.4 to 1.0. All 96 simulations were run for 2000 days. Statistics were accumulated after a statistically steady state was reached: they are averages over the last 600 days, sampled four times daily.

\section{b. Idealized moist GCM}

The idealized moist GCM is described in O'Gorman and Schneider (2008) and is similar to that of Frierson (2007). It simulates an atmosphere with an interactive hydrologic cycle, over a uniform ocean surface with a thermal inertia equivalent to $1 \mathrm{~m}$ of water. The shortwave albedo of the planet is set to a uniform value of 0.38 . Other constants (insolation, rotation rate, gravity, etc.) are kept the same as on Earth. A spectral dynamical core solves for large-scale motions in the atmosphere with a resolution of T42. This lower horizontal resolution was chosen to reduce computational time. We reran a small number of simulations at T85 resolution; we found no significant differences from the results prescribed here at this higher resolution. As in the dry GCM, the vertical coordinate is discretized with 30 sigma levels. Water vapor is advected by the flow, and it condenses whenever saturation occurs on the grid scale. Momentum, moisture, and dry entropy fluxes at the surface are parameterized by bulk aerodynamic formulas; a $k$-profile boundary layer scheme similar to Troen and Mahrt (1986) represents vertical transport by subgrid-scale motions. Moist convection is parameterized by a quasi-equilibrium convection scheme, which is like that in Frierson (2007), with the modifications described in O'Gorman and Schneider (2008). Radiative heating rates are computed for a gray atmosphere with no clouds, in which a prescribed longwave optical thickness roughly accounts for longwave absorption by water vapor and well-mixed greenhouse gases. The GCM is forced by an approximation of annual-mean insolation at the top of the atmosphere. This insolation is steady (i.e., there is no diurnal or seasonal cycle). The reference longwave optical thickness profile is chosen to lead to an extratropical climate resembling Earth's in the annual mean (O'Gorman and Schneider 2008). To obtain climate scenarios with a tropical climate and Hadley circulation resembling Earth's, it is necessary to account for ocean heat transport in low latitudes. We use a simple representation of ocean heat transport, which couples the heat transport to surface temperature gradients and surface wind stress (Klinger and Marotzke 2000; Levine and Schneider 2011).

We simulate a wide range of climates by varying the longwave optical depth and the insolation profile, in climate scenarios with and without the coupled ocean heat transport. The reference profile for the longwave optical depth is rescaled by a constant factor $\alpha$ to mimic changes in greenhouse gas concentrations; we use seven different rescaling factors, ranging from $\alpha=0.2$ 
TABLE 1. List of all configurations for moist GCM simulations (with or without ocean heat flux). Global-mean surface temperature and equator-to-pole contrast are shown in each configuration for the slab simulations. Columns show variations due to change in the rescaling coefficient of longwave optical depth. Rows show variations with insolation contrast. The reference simulation is defined by $\alpha=1.0$ and $\Delta=1.2$, which is characterized by global-mean and equator-to-pole temperature contrast close to those of Earth in the annual mean ( 287 and $49 \mathrm{~K}$, respectively, for the Southern Hemisphere).

\begin{tabular}{|c|c|c|c|c|c|c|c|c|c|c|c|c|c|c|c|c|c|c|c|}
\hline \multirow[b]{2}{*}{$\alpha$} & \multicolumn{19}{|c|}{$\Delta$} \\
\hline & 0 & 0.1 & 0.2 & 0.4 & 0.6 & 0.8 & 1.0 & 1.2 & 1.4 & 1.6 & 1.8 & 2.0 & 2.2 & 2.4 & 2.6 & 2.8 & 3.0 & 3.2 & 3.4 \\
\hline \multirow[t]{2}{*}{0.2} & 262 & 262 & 262 & 262 & 261 & 261 & 260 & 260 & 259 & 258 & 257 & 256 & 254 & 253 & - & - & - & - & - \\
\hline & 14 & 19 & 24 & 33 & 43 & 52 & 61 & 69 & 77 & 90 & 99 & 112 & 126 & 144 & - & - & - & - & - \\
\hline \multirow[t]{2}{*}{0.4} & 274 & 273 & 273 & 273 & 272 & 271 & 271 & 270 & 269 & 268 & 267 & 266 & 265 & 263 & - & - & - & - & - \\
\hline & 23 & 27 & 31 & 38 & 45 & 51 & 59 & 64 & 72 & 82 & 92 & 101 & 113 & 125 & - & - & - & - & - \\
\hline \multirow[t]{2}{*}{0.7} & 285 & 284 & 284 & 284 & 283 & 282 & 281 & 280 & 279 & 278 & 277 & 276 & 275 & 274 & - & - & - & - & - \\
\hline & 22 & 25 & 28 & 34 & 39 & 45 & 51 & 56 & 64 & 71 & 79 & 87 & 97 & 107 & - & - & - & - & - \\
\hline \multirow[t]{2}{*}{1.0} & 291 & 291 & 291 & 290 & 290 & 289 & 288 & 287 & 286 & 285 & 284 & 283 & 281 & 280 & - & - & - & - & - \\
\hline & 19 & 22 & 24 & 28 & 33 & 38 & 43 & 49 & 54 & 61 & 69 & 76 & 84 & 95 & - & - & - & - & - \\
\hline \multirow[t]{2}{*}{1.6} & 299 & 299 & 299 & 298 & 298 & 297 & 297 & 296 & 295 & 294 & 293 & 292 & 290 & 289 & 288 & 286 & 284 & - & - \\
\hline & 16 & 17 & 18 & 22 & 25 & 29 & 32 & 37 & 42 & 47 & 53 & 60 & 68 & 75 & 86 & 96 & 106 & - & - \\
\hline \multirow[t]{2}{*}{2.5} & 306 & 306 & 306 & 305 & 305 & 304 & 304 & 303 & 303 & 302 & 301 & 300 & 299 & 298 & 296 & 294 & 293 & - & - \\
\hline & 13 & 14 & 15 & 18 & 20 & 23 & 26 & 29 & 32 & 36 & 40 & 46 & 51 & 59 & 68 & 77 & 88 & - & - \\
\hline \multirow[t]{2}{*}{4.0} & 312 & 312 & 312 & 312 & 311 & 311 & 311 & 310 & 310 & 309 & 309 & 308 & 307 & 306 & 305 & 303 & 302 & 300 & 298 \\
\hline & 11 & 12 & 14 & 16 & 18 & 21 & 23 & 25 & 28 & 30 & 33 & 37 & 41 & 47 & 54 & 62 & 72 & 83 & 94 \\
\hline
\end{tabular}

to 4.0. The insolation profile is varied by varying the pole-to-equator insolation contrast, while keeping the global mean fixed. This is achieved by varying the nondimensional factor $\Delta$ in the top-of-atmosphere insolation $S_{\mathrm{TOA}}=S_{o} / 4\left[1+\Delta / 4\left(1-3 \sin ^{2} \phi\right)\right]$, where $S_{o}=1360 \mathrm{~W} \mathrm{~m}^{-2}$ is the total solar irradiance. We use between 14 and 19 insolation contrast values for every optical depth value, for a total of 109 climate simulations, each with and without coupled ocean heat transport (see Table 1 for a summary of all configurations). Some of these insolation contrast values are so large that insolation becomes negative at the poles; these simulations, despite not being realizable planetary configurations, extend our study to a climate range similarly wide as that covered by the dry GCM simulations. All simulations were run for 9 years. Statistics were accumulated over the last 4 years, from fourtimes-daily samples.

\section{c. Earth's climate}

In addition to these simulations, we analyzed Earth's annual-mean climate as given by ERA-Interim (Dee et al. 2011). The reanalysis data were averaged annually over a 30-yr period (1979-2008). To ease comparison between theory and observation, all variables were computed for the Southern Hemisphere only. This was done to mitigate added complications arising from the existence of continents and large mountain ranges in the Northern Hemisphere. Selecting the Southern Hemisphere allows for a comparison with Earth's climate under conditions more compatible with the assumptions used to derive the supercriticality and effective static stability.

\section{Results}

This ensemble of dry and moist simulations (218 moist and 96 dry simulations) spans a very wide range of climates. In the moist GCM simulations without ocean heat transport, global-mean surface temperatures vary from 253 to $312 \mathrm{~K}$, and equator-to-pole temperature contrasts vary from 11 to $144 \mathrm{~K}$ (Table 1); the ranges are essentially the same for the simulations with ocean heat transport. Among the moist simulations, we identify a reference simulation ( $\alpha=1.0$ and $\Delta=1.2$ ), which has an equator-to-pole surface temperature contrast $(49 \mathrm{~K})$ and a global-mean surface temperature $(287 \mathrm{~K})$ close to Earth's in the annual mean. In the dry GCM simulations, global-mean surface temperatures vary from 243 to $385 \mathrm{~K}$, and equator-to-pole temperature contrasts from 12 to $100 \mathrm{~K}$.

Slab and ocean moist simulations have nearly identical global means and equator-to-pole contrasts of the surface temperature. Yet the Hadley circulation was found to be sensitive to the presence of ocean heat transport in the tropics: Levine and Schneider (2011) found that the Hadley circulation can become significantly wider (by $5^{\circ}$ or more) and weaker (by $50 \%$ or more) when ocean heat transport is introduced. For example, in the reference climate ( $\alpha=1.0$ and $\Delta=1.2)$, the reference simulation with ocean heat transport is characterized by a Hadley circulation strength [109Sv 

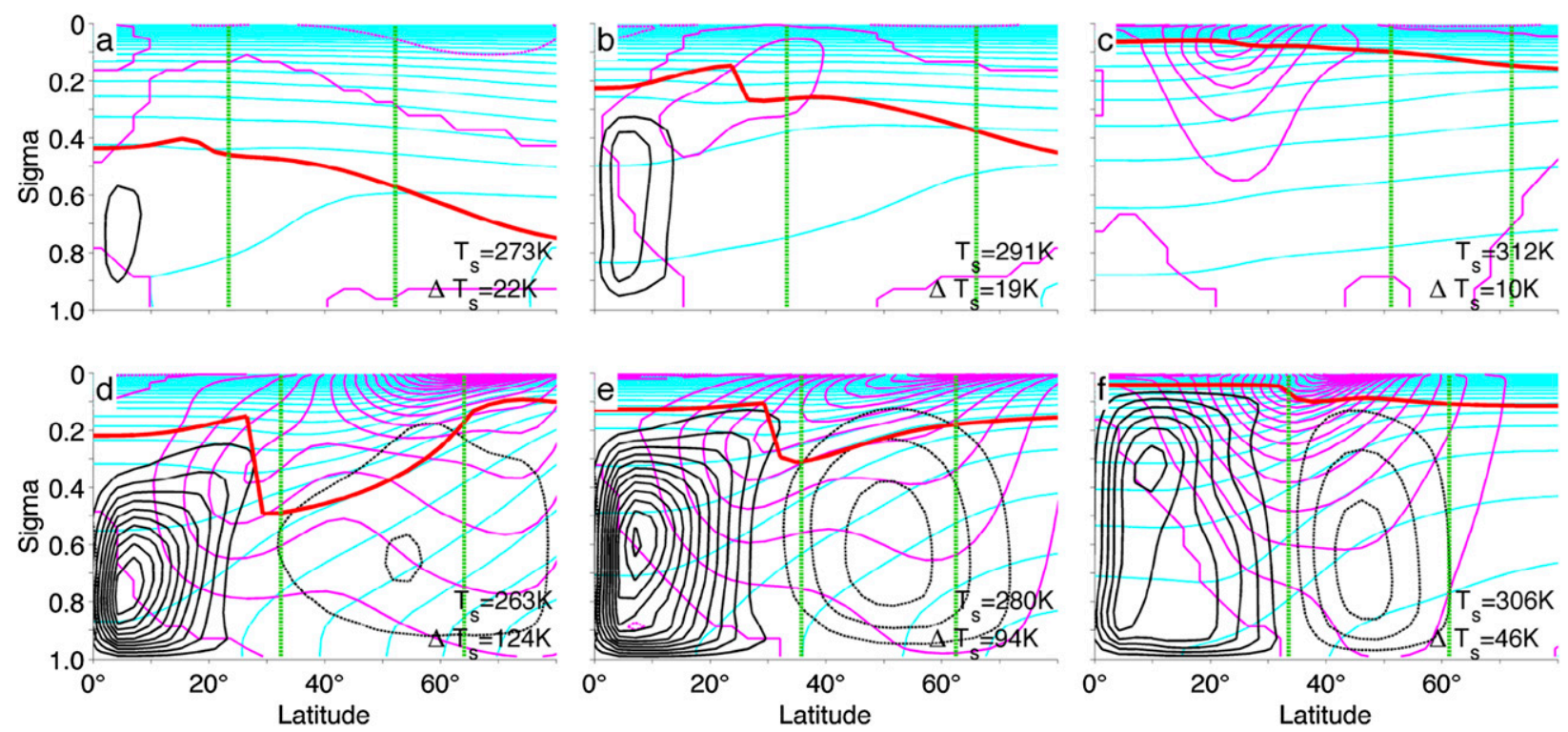

FIG. 2. Mass flux streamfunction (clockwise: solid black lines; counterclockwise: dashed black lines), mean potential temperature (cyan lines), mean zonal wind (magenta lines), and mean tropopause height (red solid line) for the following moist GCM simulations (without ocean heat transport): (a) a cold climate with low equator-to-pole temperature contrast $\Delta T_{s}$, (b) a temperate climate with low $\Delta T_{s}$, (c) a warm climate with low $\Delta T_{s}$, (d) a cold climate with large $\Delta T_{s}$, (e) a temperate climate with large $\Delta T_{s}$, and (f) a warm climate with large $\Delta T_{s}$. The poleward and equatorward boundaries of the baroclinic zone, as defined in appendix A, are shown by the vertical green dashed lines. The global-mean surface temperature and the equator-to-pole temperature contrast of these simulations are shown in the bottom-right corner of each panel. Contour intervals are $10 \mathrm{~m} \mathrm{~s}^{-1}$ for zonal wind, $20 \times 10^{9} \mathrm{~kg} \mathrm{~s}^{-1}$ for streamfunction, and $10 \mathrm{~K}$ for potential temperature.

$\left.\left(1 \mathrm{~Sv} \equiv 106 \mathrm{~m}^{3} \mathrm{~s}^{-1}\right)\right]$ and width $\left(26^{\circ}\right)$ comparable to Earth's in the annual mean $\left(111 \mathrm{~Sv}\right.$ and $30^{\circ}$, respectively). By contrast, the reference simulation without ocean heat transport has an unrealistically strong $(181 \mathrm{~Sv})$ and narrow $\left(23^{\circ}\right)$ Hadley circulation. Ocean heat transport becomes increasingly important for the Hadley circulation as the global-mean surface temperature decreases or its equator-to-pole contrast increases.

As the global-mean surface temperature increases, the tropopause shifts upward (Fig. 2), consistent with the radiative-convective response of an atmosphere to an increase in longwave opacity (Thuburn and Craig 2000; Schneider 2007). Increasing the equator-to-pole temperature contrast also deepens the troposphere through an increase in static stability that, other factors equal, usually accompanies increased meridional temperature contrasts. The increase in tropopause height with static stability can likewise be understood from radiative constraints on the tropopause (Held 1982; Schneider 2007).

\section{a. Supercriticality in midlatitudes}

Schneider and Walker (2006) found $S_{c} \lesssim 1$ in baroclinic zones of dry GCM simulations, with $S_{c}<1$ when convection played a substantial role in stabilizing the extratropical thermal stratification. Here, we address whether the effective supercriticality, which takes the stabilizing effect of convection in baroclinic eddies into account, leads to a scaling $S_{c}^{\text {eff }} \simeq 1$ over a wider range of climates in the dry GCM simulations and can also account for latent heat release in baroclinic eddies in the moist GCM simulations.

Figure 3 shows the traditional static stability (3) versus the meridional potential temperature contrast (2) in all dry and moist GCM simulations. Simulations with $S_{c}=1$ lie on the one-to-one line. Colors indicate the convective static stability, evaluated from the static stability (3) using the convective lapse rate and tropopause pressure and averaged over the baroclinic zones for each GCM simulation (see appendix A for averaging conventions). Dry or moist simulations with (nearly) dry adiabatic convective lapse rates lie near the line $S_{c}=1$ (dark blue colors in Fig. 3). Moist simulations with nearly dry adiabatic convective lapse rate are cold climates. In them, the convective static stability is nearly zero, and the thermal stratification is stabilized by essentially dry baroclinic eddies. However, these essentially dry simulations in which latent heat effects are negligible cover only a small subset of all simulations. For example, among the moist simulations, only those with global-mean surface temperatures below $270 \mathrm{~K}$ fall into this category. Most simulations, including the Earth-like reference simulations with the moist GCM, lie above the one-to-one 

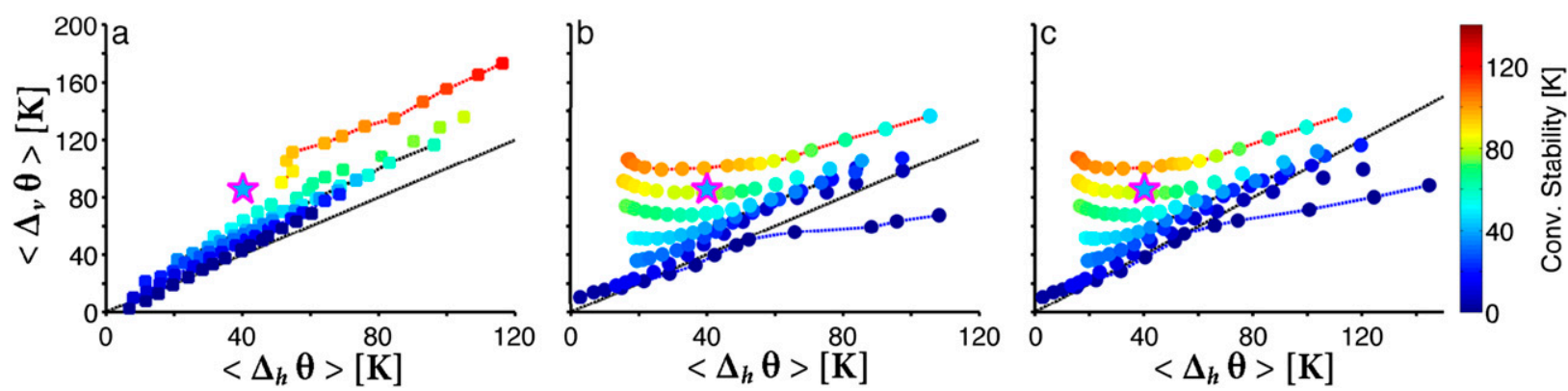

FIG. 3. Meridional potential temperature contrast (2) and static stability (3) in (a) 89 dry simulations, (b) 109 moist simulations with a slab ocean, and (c) 109 moist simulations with ocean heat transport. Colors indicate the convective static stability, averaged over the baroclinic zones for each GCM simulation (see appendix A for averaging conventions). Earth's Southern Hemisphere climate in the annual mean is marked by a pentagram. The black dashed line shows the one-to-one line (i.e., $\left.S_{c}=1.0\right)$. Dashed lines in (a) show a series of dry GCM simulations with varying equator-to-pole surface temperature contrast in radiative equilibrium and fixed convective lapse rates $\Gamma=\gamma \Gamma_{d}$, where $\Gamma_{d}$ is the dry adiabatic lapse rate (blue: $\gamma=0.9$; black: $\gamma=0.7$; red: $\gamma=0.6$ ). Dashed lines in (b) and (c) show a series of moist GCM simulations with varying equator-to-pole insolation contrast and fixed longwave opacity profile $\alpha \tau_{\text {ref }}^{\text {lw }}$, where $\tau_{\text {ref }}^{\text {lw }}$ is the Earth like reference optical depth (blue: $\alpha=0.2$; black: $\alpha=1.0$; red: $\alpha=4.0$ ). The extratropical thermal stratification in simulations clustered on the one-to-one line is controlled by baroclinic eddies, while in those clustered above the black line (i.e., $S_{c} \leq 1$ ), it is controlled by radiative-convective processes.

line in Fig. 3. ${ }^{1}$ Figure 3 also suggests that Earth's climate lies above the one-to-one line. In these simulations, as well as in Earth's atmosphere, the static stability (3) is larger than the meridional potential temperature contrast (2) (i.e., $S_{c}<1$ ). Generally, $S_{c}$ decreases as the convective lapse rate decreases (i.e., as colors in Fig. 3 shift from blue to red). A value of $S_{c}<1$ implies that convection plays a role in stabilizing the extratropical thermal stratification, although it may not act alone but in conjunction with baroclinic eddies, because the static stability is typically greater than that implied by convection alone (cf. Schneider and Walker 2006). For example, moist simulations with large meridional temperature contrasts are characterized by static stability values around $160 \mathrm{~K}$ (ordinate of Fig. 3). This exceeds the convective static stability, which would be less than $120 \mathrm{~K}$ (colorbar in Fig. 3). In these simulations, the supercriticality does not provide information on the extratropical thermal stratification beyond the inequality constraint $S_{c} \lesssim 1$, and it does not discriminate effectively between purely convective and baroclinic-convective static stability regimes.

Using the effective static stability (10), with the specified convective lapse rate in the case of the dry simulations, better characterizes dynamical regimes in

\footnotetext{
${ }^{1}$ Seven dry GCM simulations with low meridional potential temperature contrasts and low convective lapse rates were dominated by numerical noise. This prevented a robust estimate of baroclinic zones and of the Hadley circulation terminus. They were excluded from Fig. 3 and all subsequent figures. In them, the tropopause height is controlled by convection.
}

the extratropics. Figure 4 shows the effective static stability (10) versus the meridional potential temperature contrast (2). Comparing Figs. 3 and 4, we see that now a much larger fraction of the simulations, both moist and dry, lie on the one-to-one lines that signify $S_{c}^{\text {eff }}=1$. Only in moist GCM simulations with weak meridional temperature contrasts does convection control the extratropical thermal stratification $\left(S_{c}^{\mathrm{eff}}<1\right)$. All other simulations characterized by $S_{c}^{\text {eff }}=1$ have greater effective static stability than predicted from purely convective adjustment, as shown by comparing the ordinate to the values denoted by the colorbar in Fig. $4 .^{2}$ In the moist simulations, the transition from baroclinic to convective regimes is temperature dependent, requiring greater baroclinicity as the climate warms: while the coldest moist simulations are controlled by baroclinic eddies regardless of their baroclinicities, in the warmest simulations, baroclinic eddies only dominate the extratropical thermal stratification when the meridional potential temperature contrast becomes larger than around $50 \mathrm{~K}$, which is when the meridional potential temperature contrast equals the effective static stability in radiative-convective equilibrium. Thus, the effective supercriticality can provide information on the extratropical thermal stratification

\footnotetext{
${ }^{2}$ Figure 4 shows four moist GCM simulations below the one-toone line (i.e., with $S_{c}^{\text {eff }}>1$ ). These simulations correspond to very cold climates, with near-neutral temperature lapse rates. These simulations appear to be consistent with the findings of Schneider and Walker (2006) and Jansen and Ferrari (2012), who also found $S_{c}>1$ in simulations with near-neutral lapse rates.
} 


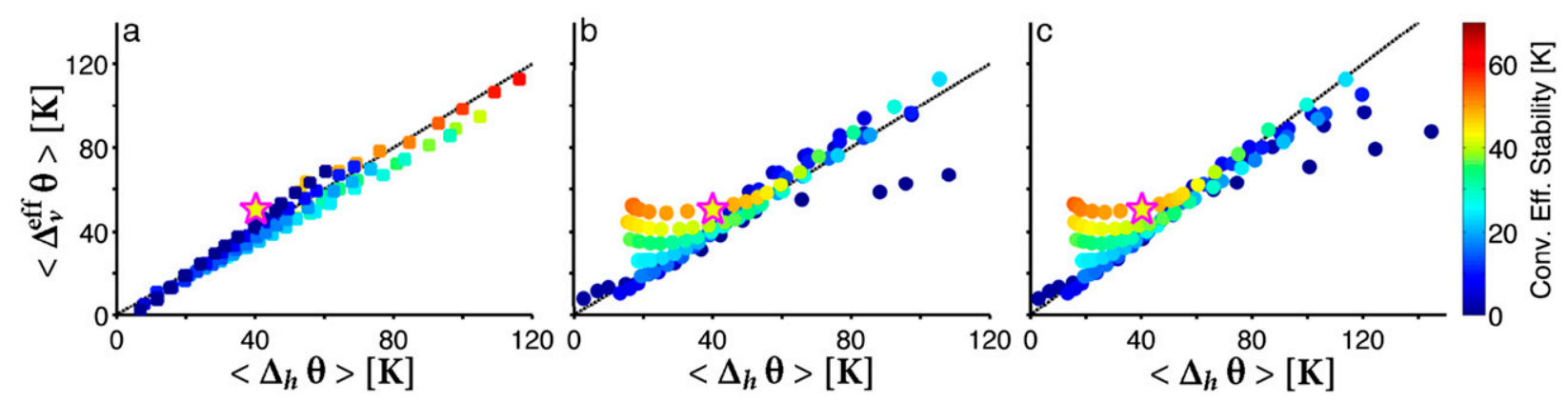

FIG. 4. Meridional potential temperature contrast (2) and effective static stability (10) for the simulations shown in Fig. 3. Colors indicate the convective effective static stability averaged over the baroclinic zones for each GCM simulation. Other plotting conventions are as in Fig. 3.

even when convection and/or large-scale latent heat release in baroclinic eddies are important for the extratropical thermal stratification, as suggested by O'Gorman (2011) for moist simulations similar to those here. It can discriminate more effectively between purely convective and baroclinic-convective static stability regimes. Comparison of Figs. 3 and 4 shows that convection influences how baroclinic eddies affect the thermal stratification, even when turbulence is strong or when large-scale latent heat release has no effect on the dynamics. Comparing these results to Earth's climate, Fig. 4 suggests that Earth's annual-mean climate is marginally subcritical, with an effective supercriticality value of about 0.8 in the annual mean for the Southern Hemisphere. This finding appears consistent with Korty and Schneider (2007), who found convective adjustment to be a dominant process in Earth's extratropical troposphere in some regions and seasons (e.g., in summer over continents and in winter over oceanic storm tracks).

Comparing Figs. $4 \mathrm{~b}$ and $4 \mathrm{c}$, we see that moist simulations with and without parameterized ocean heat flux show similar behavior. Despite its large effect on the dynamics of the tropical atmosphere, ocean heat transport in the tropics has little effect on the extratropical atmosphere (Levine and Schneider 2011).

\section{b. Extent of Hadley circulation}

Despite a large body of theoretical and observational studies, there is no unique definition for the terminus of the Hadley circulation (cf. Levine and Schneider 2011). Here, we define the terminus as the subtropical latitude where the mean meridional mass flux, integrated from the 750-hPa pressure level to the top of the atmosphere, attains $10 \%$ of its tropical extremum. In most climates, the 750-hPa pressure level is near the upper bound of the lower branch of the Hadley circulation. This definition is similar to others used in the literature (e.g., Lu et al. 2007). Figure 5 shows that the latitude of the terminus in both moist and dry simulations varies widely, from $8^{\circ}$ to $36^{\circ}$. Using slightly different definitions for the terminus based on the mass flux streamfunction (for instance, the latitude of its zero crossing) does not change our results significantly. Dry GCM simulations suggest the existence of a maximum width for the Hadley circulation on an Earth-like planet: in simulations with large convective static stabilities and large meridional potential temperature contrasts, the terminus appears to asymptotically approach $36^{\circ}$. This maximum extent is generally reached in simulations with large baroclinicity. One might think this maximum latitude is given by the HeldHou extent of angular momentum-conserving Hadley circulations [see (C1)]. However, unlike the angular momentum-conserving extent, the terminus latitude in the dry simulations depends on the static stability, and it does not appear to continue to increase as the meridional potential temperature contrast increases.

In the moist simulations, the Hadley circulation widens as the climate warms, in agreement with comprehensive global warming simulations (e.g., Lu et al. 2007; Medeiros et al. 2015). The Hadley circulation terminus moves from $16^{\circ}$ to $27^{\circ}$ when the longwave opacity in the simulations increases, while the insolation is kept fixed to Earth's annual-mean profile (dashed lines in Fig. 5). The terminus also shifts poleward as the insolation contrast, and with it meridional temperature contrasts, increase: when the longwave opacity is kept fixed to its reference profile while the insolation contrast is increased from zero to its maximum value, consistent with nonnegative insolation at the poles, the terminus moves from $16^{\circ}$ to $26^{\circ}$ (i.e., simulations on Fig. 5, denoted by a green color, shifting from the dashed red line to the dashed blue line as insolation contrast increases). In the reference simulation without ocean heat transport, the Hadley circulation extends to $23^{\circ}$. This is a $7^{\circ}$ smaller extent than that of Earth's Hadley circulation in the annual mean. Once ocean heat transport is included, 

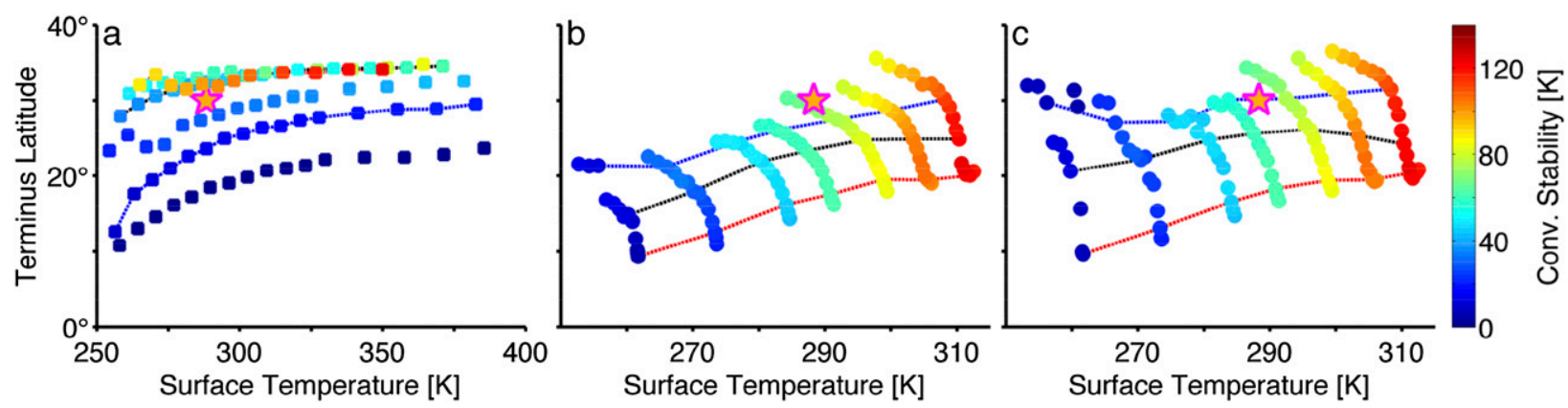

FIG. 5. Latitude of the terminus of the Hadley circulation and global-mean near-surface temperature for simulations shown in Fig. 3. Colors indicate the convective static stability, evaluated locally at the latitude of the terminus. (a) As in Fig. 3, dashed lines show a series of dry GCM simulations with varying equator-to-pole surface temperature contrast in radiative equilibrium and with fixed convective lapse rates (blue: $\gamma=0.9$; black: $\gamma=0.7$; red: $\gamma=0.6$ ). (b),(c) Dashed lines show a series of moist GCM simulations with varying longwave opacity and fixed equator-to-pole insolation contrast (blue: $\Delta=2.0$; black: $\Delta=1.2$; red: $\Delta=0.2$ ). Other plotting conventions are as in Fig. 3.

the Hadley circulation in the reference simulation is $26^{\circ}$ wide and closer to Earth's in the annual mean (cf. Figs. 5b,c), consistent with the results of Levine and Schneider (2011).

In the dry simulations, Fig. 5 shows that the terminus shifts poleward as the equator-to-pole contrast in radiative equilibrium increases or the convective lapse rate decreases. These variations are qualitatively consistent with a widening of the circulation that accompanies a deepening of the troposphere or an increase in extratropical baroclinicity, as seen in the moist GCM simulations and in observations (Adam et al. 2014).

\section{c. Supercriticality at the terminus of the Hadley circulation}

In the simulations in which the extratropical thermal stratification is baroclinically controlled and $S_{c}^{\text {eff }} \approx 1$, the terminus of the Hadley circulation sits at the latitude at which $S_{c}^{\text {eff }}$ first exceeds a critical $O(1)$ value. That is, to the extent the effective supercriticality is a good measure of the depth of baroclinic eddies, the Hadley circulation extends to the latitude where baroclinic eddies become deep enough to reach the upper troposphere. This can be seen in Fig. 6, which shows the effective static stability (10) versus the meridional potential temperature contrast (2) evaluated at the latitude of the terminus. Comparing these local quantities with the bulk quantities estimated over baroclinic zones (Fig. 4), we find very similar results. The only noticeable difference is the slope of the line around which simulations cluster. When baroclinic eddies control the extratropical thermal stratification (i.e., $S_{c}^{\text {eff }} \approx 1.0$ over the baroclinic zones), a lower but largely invariant value of supercriticality is found at the terminus $\left(S_{c}^{\text {eff }} \approx 0.7\right)$. This lower value of the supercriticality is conceptually consistent with the terminus being a transition between regions where baroclinic eddies dominate the dynamics (i.e., extratropical storm tracks) and regions where convection exerts a direct control on the tropospheric thermal stratification (i.e., the tropics). With the interpretation of Schneider and Walker (2006), $S_{c}^{\text {eff }} \approx 0.7$ also implies that baroclinic eddies generated at the latitude of the terminus extend over approximately $70 \%$ of the extratropical tropospheric depth. Simulations found in the convective regime are characterized by $S_{c}^{\text {eff }}<0.7$. This also seems to apply to Earth's climate (Fig. 6), in which the effective supercriticality was estimated to be about 0.36 in the annual mean at the terminus of the Hadley cell in the Southern Hemisphere. This is in agreement with convection exerting some influence on the thermal stratification of the extratropical troposphere.

Similar to what we found over the baroclinic zones, using the dry static stability in lieu of the effective static stability significantly degrades the ability of the supercriticality to predict the latitude of the Hadley circulation terminus. That is, accounting for convection and large-scale condensation is as important near the terminus as it is over the storm tracks.

To the extent that $S_{c}^{\text {eff }} \approx 0.7$ holds at the terminus, the Hadley circulation would be expected to be wider in the ocean simulations than in the corresponding slab simulations, as is indeed seen in the simulations. The reason is that ocean heat transport warms the subtropics and cools the deep tropics, thereby reducing the meridional surface temperature gradient in the tropics and subtropics. Lower baroclinicity on the equatorward side of the Hadley circulation terminus is compensated by the terminus being shifted poleward in the ocean simulations. 


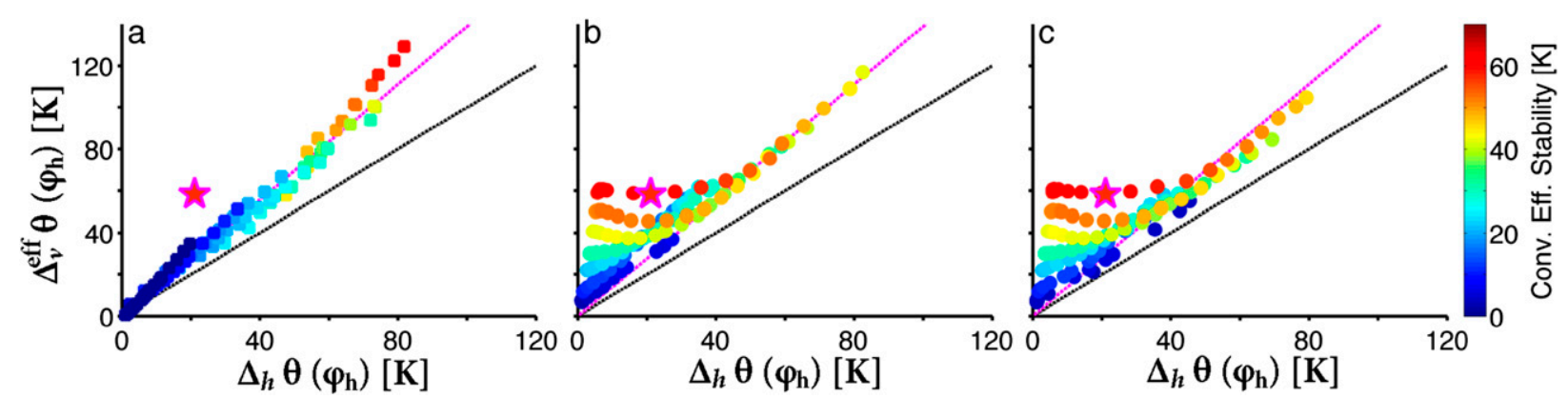

FIG. 6. Meridional potential temperature contrast (2) and effective static stability (10) evaluated at the latitude of the Hadley circulation terminus using the same simulations and plotting conventions as in Fig. 5. The magenta lines correspond to $S_{c}^{\text {eff }}=0.7$.

\section{Discussion}

In our simulations, the Hadley circulation was found to widen with global warming, associated with an increase in static stability and a lifting of the extratropical tropopause height. This widening with global warming accompanies a poleward shift of the storm tracks (Schneider 2004; Lorenz and DeWeaver 2007; Mbengue and Schneider 2013). For example, increasing the longwave optical depth of the atmosphere by a factor of 30 in moist simulations leads to a $10^{\circ}$ shift of the terminus, from $15^{\circ}$ to $25^{\circ}$; the storm tracks shift as much, from $44^{\circ}$ to $54^{\circ}$. Both shifts appear to be caused by the increase in tropospheric latent heating (increasing static stability) and lifting of the tropopause associated with the global warming. Previous studies using comprehensive GCM simulations have also recognized that the widening of the Hadley circulation is associated with an increase in static stability (Lu et al. 2008), a lifting of the extratropical tropopause height (Lu et al. 2007), or a decrease in the wind shear (Tandon et al. 2013). Our study quantifies this effect using the invariance of the effective supercriticality at the latitude of the terminus.

The Hadley circulation was generally found to widen when the equator-to-pole temperature contrast increased in both moist and dry GCM simulations. In this scenario, the subtropical static stability was found to increase faster than the local meridional potential temperature contrast, and this leads to a poleward shift of the terminus. This is the opposite of what happens during an El Niño, when an anomalous warming of the tropical troposphere (and thus an increase in the equator-to-pole temperature gradient) leads to a contraction of the Hadley circulation. In the latter case, anomalous heating in the deep tropics increases the meridional temperature gradient in the subtropics, but without large compensation from a static stability increase, and this leads to an equatorward shift of the terminus. Our results are consistent with the findings of Brayshaw et al. (2008), who showed that the storm tracks (and perhaps also the Hadley circulation terminus) could shift poleward or equatorward when increasing baroclinicity (e.g., by prescribing an anomalous SST gradient), depending on whether this anomalous baroclinicity is applied in the subtropics (in which case storm tracks shift equatorward) or in the midlatitudes (in which case storm tracks shift poleward).

It is also important to note that the terminus of the Hadley circulation and the storm-track latitude are not equally sensitive to climate changes, depending on which forcings are varied (Mbengue and Schneider 2013). For instance, increasing the insolation contrast from $\Delta=0$ to $\Delta=2.0$ in simulations with the referencelongwave optical depth $(\alpha=1.0)$ induces an $11^{\circ}$ shift of the terminus, from $16^{\circ}$ to $27^{\circ}$, and this outpaces the $2^{\circ}$ shift of the latitude of the storm tracks. This difference comes from the different sensitivity of the static stability in the subtropics and extratropics: while the increase in meridional temperature gradient is matched by static stability increases when averaged over the baroclinic zones, static stability in the subtropics increases more rapidly. The decoupling of the isentropic slope (i.e., the ratio of the meridional potential gradient and static stability) between the subtropics and the extratropics in these simulations calls into question whether changes in the isentropic slopes in the subtropics generally translate into shifts in the storm tracks, as suggested by Butler et al. (2011).

\section{Conclusions}

Using a dry and a moist idealized GCM, we have simulated a wide range of climates. In the simulations, the terminus of the Hadley circulation varied between about $8^{\circ}$ and $36^{\circ}$. A supercriticality criterion was modified to account for the tight coupling between baroclinic eddies and convection. This effective supercriticality 
criterion successfully discriminated between simulations in which the extratropical thermal stratification was controlled by baroclinic eddies and those in which it is controlled locally by convection. The latitude of the Hadley circulation terminus was found to be well constrained by a constant $O(1)$ value of the effective supercriticality whenever baroclinic eddies were strong; in this regime, the Hadley circulation terminus acts as the equatorward boundary for baroclinic wave activity that reaches the upper troposphere, as suggested in the dry context by Korty and Schneider (2008).

We found that the extratropical thermal stratification in idealized moist GCM simulations is controlled by baroclinic eddies when the meridional potential temperature contrast becomes larger than the effective static stability in local convective equilibrium. The Earth-like reference climate was found to be near the transition between baroclinic and convective regimes. Earth's Southern Hemisphere climate in the annual mean similarly shows an important influence from convection on the thermal stratification. More generally, we found convection to influence the static stability and tropopause height, even when baroclinic eddies were strong.

The finding that the effective supercriticality typically assumes a constant $O(1)$ value near the Hadley circulation termini may offer an explanation for why the Hadley cells contract under El Niño but expand under La Niña and global warming (Seager et al. 2003; Lu et al. 2008; Tandon et al. 2013; Adam et al. 2014). Under El Niño, the equatorial atmosphere warms, leading to increased equator-to-pole temperature contrasts in the lower troposphere (e.g., Seager et al. 2003). For $S_{c}$ to assume a constant value at the Hadley circulation termini, the Hadley circulation needs to contract, provided changes in static stability in the lower troposphere are not large enough to compensate. Conversely, reduced pole-to-equator temperature contrasts under La Niña demand an expansion of the Hadley circulation. ${ }^{3}$ Similarly, under global warming, the static stability and tropopause height near the Hadley circulation termini generally increase, both ultimately because surface

\footnotetext{
${ }^{3}$ The relevance of supercriticality to ENSO events is difficult to assess quantitatively from observations, as the Northern Hemisphere Hadley circulation contracts by less than $2^{\circ}$ during a typical El Niño event, with significant discrepancies among observational datasets (e.g., Nguyen et al. 2013). But prescribing a heat source in the deep tropics in our idealized moist GCM induces a contraction of the Hadley circulation as during an El Niño event, and the effective supercriticality criterion captures these changes (T. Bischoff 2014, personal communication). These results will be described in a forthcoming publication.
}

temperatures increase. This leads to increased static stability because of increased latent heat release (e.g., Frierson et al. 2006; Schneider and O'Gorman 2008) and an elevated tropopause because of higher temperatures and increased static stability (e.g., Schneider 2007). Overall, these conclusions are qualitatively consistent with previous studies, which found the Hadley circulation to widen in response to a global (or extratropical) heating but to contract in response to a tropical heating (Butler et al. 2011; Tandon et al. 2013). The effective supercriticality criterion for the extent of the Hadley circulation affords a unified and simple interpretation of these seemingly disparate phenomena.

Acknowledgments. We thank Paul O'Gorman for helpful clarifications on the effective static stability, Timothy M. Merlis for discussions on both linear baroclinic wave theories and ocean-atmosphere interactions, and Tobias Bischoff for his helpful comments on this work and its relation to ENSO. We are grateful for support by the National Science Foundation (Grants AGS1019211 and AGS-1049201) and a Yale Climate and Energy Institute Fellowship. The simulations were performed on the Division of Geological and Planetary Sciences' Dell cluster at the California Institute of Technology (the program code for the simulations described in this paper is available at www.clidyn.ethz.ch/gcms/).

\section{APPENDIX A}

\section{Determination of Baroclinic Zones}

The supercriticality may be evaluated either locally (1) or as a bulk average. Schneider and Walker (2006) argued that the supercriticality should be estimated over a baroclinic zone that is at least as wide as the largest baroclinic eddies. Following Schneider and Walker (2006), we define baroclinic zones as regions where the near-surface eddy meridional potential temperature (heat) flux is greater than $50 \%$ of its maximum value. Near-surface averaging is defined as a massweighted vertical averaged between sigma levels 0.75 and 0.85. On an Earth-like planet (in size and rotation rate), this region covers most of the extratropics, consistent with the deformation radius being of the scale of the planet. Schneider and Walker (2006) defined the center of the storm tracks as the latitude where the eddy heat flux is maximum. In some climates, the eddy heat flux does not have a sharply defined maximum, which leads to sensitivity to the averaging convention when estimating baroclinic zones. We address this issue by defining the center of the storm track $\varphi_{M}$ from the eddy heat flux cumulative distribution: that is, 


$$
\varphi_{M}=\frac{\int_{\varphi_{-}}^{\varphi_{+}}\left(\overline{v^{\prime} \theta^{\prime}}\right) \varphi d p}{\int_{\varphi_{-}}^{\varphi_{+}}\left(\overline{v^{\prime} \theta^{\prime}}\right) d p} .
$$

Here, $\varphi_{-}$and $\varphi_{+}$define, respectively, the equatorward and poleward boundaries of the baroclinic zones: that is, the latitudes where the near-surface eddy meridional potential temperature (heat) flux equals $50 \%$ of its maximum value, equatorward and poleward of the latitude of its maximum value. In a moist GCM simulation characterized by an Earth-like climate (e.g., $\Delta=1.2$ and $\alpha=1.0$ ), we found $\varphi_{-}=28^{\circ}, \varphi_{+}=68^{\circ}$, and $\varphi_{M}=48^{\circ}$ in each hemisphere. Generally, the width of the baroclinic zones stays relatively invariant with climate changes. Yet storm-track latitudes (as well the boundaries of the baroclinic zones) can shift with climate changes.

A bulk estimate of supercriticality can then be defined as

$$
\left\langle S_{c}\right\rangle=\frac{\left\langle\Delta_{h} \theta\right\rangle}{\left\langle\Delta_{v} \theta\right\rangle}
$$

where $\left\langle\Delta_{h} \theta\right\rangle$ is defined as the bulk meridional potential temperature contrast,

$$
\left\langle\Delta_{h} \theta\right\rangle=-\frac{f\left(\varphi_{M}\right)}{\beta\left(\varphi_{M}\right)}\left[\partial_{y} \bar{\theta}_{s}\right],
$$

and $\left\langle\Delta_{v} \theta\right\rangle$ as the bulk static stability:

$$
\left\langle\Delta_{v} \theta\right\rangle=-2\left[\partial_{p} \bar{\theta}_{s}\right]\left[\bar{p}_{o}-\bar{p}_{t}\right] .
$$

Here, the angle braces indicate a bulk estimate, the square brackets define a meridional average over the baroclinic zone, and $\bar{p}_{o}$ is the reference pressure at the surface $\left(\bar{p}_{o}=10^{5} \mathrm{~Pa}\right)$. Similarly, a bulk convective static stability can be defined as

$$
\left.\left\langle\Delta_{v} \theta\right\rangle\right|_{\theta_{o}^{*}}=-2\left[\partial_{p} \overline{\left.\theta_{s}\right|_{\theta_{o}^{*}}}\right]\left[\bar{p}_{o}-\bar{p}_{t}\right],
$$

and both together determine the effective static stability.

\section{APPENDIX B}

\section{Determination of Tropopause Height}

A reliable determination of the tropopause height is essential when estimating the supercriticality in our simulations. The World Meteorological Organization (WMO) defines the tropopause as the lowest level of the atmosphere where the lapse rate is equal to or lower than a threshold lapse rate of $2 \mathrm{~K} \mathrm{~km}^{-1}$. We applied this definition to the bulk lapse rate (averaged over the baroclinic zones) for all climates. Tropopause heights estimated from this definition were then used to define the supercriticality (1). The same definition was used to define the local tropopause height in Fig. 2. We found that simulations with near-neutral convective lapse rate, which corresponds to cold climates in the moist GCM, have a poorly defined tropopause when applying the WMO definition. To circumvent this issue, we use a different definition of the tropopause height based on the meridional circulation structure.

In the coldest set of simulations, the tropopause height was redefined as the level where the mean meridional mass flux in the upper troposphere reaches its maximum value in the Northern Hemisphere. This maximum is always achieved in the deep tropics, and its latitude usually defines the center of the Hadley circulation in the Northern Hemisphere. This level usually corresponds to the levels of both maximum eddy momentum flux divergence in the tropics and convergence in the extratropics, consistent with quasi-horizontal propagation of eddies from the storm tracks to the subtropics (Ait-Chaalal and Schneider 2015). The height obtained from this definition is nearly identical to that provided by the lapse rate definition, except in the climates with near-neutral thermal stratification. In the latter simulations, the lapse rate definition fails to estimate the tropopause height because of the existence of strong inversions between the boundary layer and the free troposphere, while our dynamical definition gives a more robust estimate.

\section{APPENDIX C}

\section{Other Theories for Hadley Cell Terminus}

Held and Hou (1980) derived a relation describing the extent of an angular momentum-conserving Hadley circulation, which in the small-angle approximation is

$$
\varphi_{\mathrm{HH} 80}=\left(\frac{g H_{t}}{\Omega^{2} a^{2}} \frac{\Delta_{h} \theta_{R}}{\theta_{0}}\right)^{1 / 2} .
$$

Here, $g$ is the gravitational acceleration, $\Omega$ is the angular velocity of the planetary rotation, $a$ is the planetary radius, and $\theta_{0}$ is a constant reference value of potential temperature. Radiative-equilibrium temperatures near the equator are assumed to decrease quadratically with latitude, and $\Delta_{h} \theta_{R}$ is the equator-to-pole radiativeequilibrium temperature difference vertically averaged over the depth of the troposphere; $H_{t}$ is the height of the 

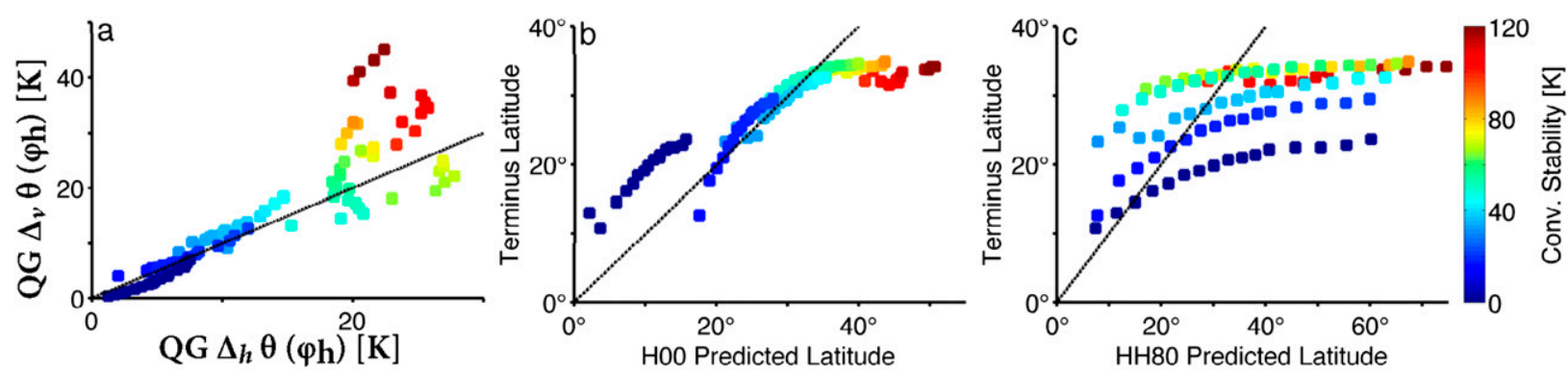

FIG. C1. Dry GCM simulations: (a) Meridional potential temperature contrast (C6) and static stability (C7), as defined in accordance to the two-layer QG criticality criterion (C5) and evaluated at the latitude of the Hadley circulation terminus. (b) Latitude of the terminus and its predicted value (C4) for an axisymmetric circulation bounded by regions of baroclinic instability. (c) Latitude of the terminus and its predicted value $(\mathrm{C} 2)$ for an axisymmetric angular momentum-conserving circulation. Colors indicate the static stability contrast in radiative-convective equilibrium evaluated locally at the latitude of the terminus. Other plotting conventions are as in Fig. 5.

upper branch of the Hadley circulation (i.e., approximately the tropical tropopause height).

We test an approximate form of relation (C1) in the dry GCM simulation, applicable to simulations with invariant planetary radius and rotation rate:

$$
\varphi_{\mathrm{HH} 80} \sim\left(H_{t} \Delta_{h} \theta_{R}\right)^{1 / 2} .
$$

Here, $H_{t}$ is averaged over the deep tropics $\left(|\phi| \leq 5^{\circ}\right)$; $\Delta_{h} \theta_{R}$ is evaluated by taking the difference between the equator and $50^{\circ} \mathrm{N}$ in the vertically averaged potential temperature profile $\theta_{R}$, obtained by running the GCM with independent atmospheric columns (without advection). Figure $\mathrm{C} 1 \mathrm{c}$ shows scaling $(\mathrm{C} 2)$ normalized by the latitude of the Hadley circulation terminus in a reference dry GCM simulation $\left(\Delta_{h}=120 \mathrm{~K}, \gamma=0.7\right)$ with Earth-like values for static stability and equatorto-pole temperature contrast in dynamical equilibrium. Figure $\mathrm{C} 1 \mathrm{c}$ shows that scaling $(\mathrm{C} 2)$ poorly predicts changes in the latitude of the Hadley circulation terminus. The terminus latitude is not nearly as sensitive to changes in the equator-to-pole temperature contrast in radiative equilibrium as predicted from the scaling (Schneider and Walker 2006). Even when baroclinic eddies are weak and the upper-tropospheric momentum balance is near its axisymmetric limit, eddy meridional heat transport usually induces large discrepancies between predicted and actual latitudes of the terminus.

Held (2000) derived a relation describing the extent of an angular momentum-conserving Hadley circulation terminated by linear baroclinic instability:

$$
\varphi_{\mathrm{H} 00}=\left(\frac{b}{2} \frac{g H_{t}}{\Omega^{2} a^{2}} \frac{\Delta_{v} \theta}{\theta_{0}}\right)^{1 / 4} .
$$

In accordance with Korty and Schneider (2008), $b$ is a constant factor, and $\Delta_{v} \theta$ is the same measure of static stability (3) as that defined for the dry supercriticality.

We test an approximate form of relation (C3) in the dry GCM simulations:

$$
\varphi_{\mathrm{H} 00} \sim\left(H_{t} \Delta_{v} \theta\right)^{1 / 4} .
$$

Here, $\Delta_{v} \theta$ defines the bulk static stability. The values of $\Delta_{v} \theta$ and $H_{t}$ are averaged over the deep tropics (i.e., $|\phi| \leq 5^{\circ}$ ). A number of studies have claimed that scaling (C4) can describe changes in the latitude of the Hadley circulation terminus, either in comprehensive GCM simulations of global warming scenarios ( $\mathrm{Lu}$ et al. 2007), or in idealized GCM simulations forced by prescribed surface temperature profiles (Frierson et al. 2007). Yet the relevance of relation (C4) to Earth's dynamics is questionable (e.g., Zurita-Gotor and Lindzen 2007). In addition, Korty and Schneider (2008) showed that relation $(\mathrm{C} 4)$ does not describe changes of the terminus latitude when tested over a large set of dry GCM simulations similar to those shown in this study. Figure $\mathrm{C} 1 \mathrm{~b}$ shows scaling $(\mathrm{C} 4)$ normalized by the latitude of the Hadley circulation terminus in the reference dry GCM simulation $\left(\Delta_{h}=120 \mathrm{~K}, \gamma=0.7\right)$. In agreement with the latter study, Fig. C1b shows that changes in the terminus latitude are not well captured by relation (C4) in dry GCM simulations forced by large equator-to-pole temperature contrasts. Yet our findings remain qualitatively consistent with $\mathrm{Lu}$ et al. (2007) and Frierson et al. (2007) for relatively small climate changes, for which simulations obey relation (C4) relatively well (see Fig. C1).

While the angular momentum-conserving regime can provide analytical predictions for the terminus latitude, it neglects the important role played by extratropical eddies in driving of the Hadley circulation (Levine and Schneider 2011). To address this issue, $\mathrm{Lu}$ et al. (2008) and Tandon et al. (2013) have investigated 
the relevance of a discretized version of the Phillips criterion for diagnosing the terminus latitude in equilibrated flows in both idealized and comprehensive GCM simulations:

$$
S_{\mathrm{QG}}=\frac{f^{2}\left(u_{500}-u_{850}\right) \theta_{0}}{\beta g H_{t}\left(\theta_{500}-\theta_{850}\right)}=\frac{\Delta_{h} \theta}{\Delta_{v} \theta},
$$

with

$$
\Delta_{h} \theta=\frac{f^{2}\left(u_{500}-u_{850}\right) \theta_{0}}{\beta g H_{t}}
$$

and

$$
\Delta_{v} \theta=\left(\theta_{500}-\theta_{850}\right)
$$

Here, $S_{\mathrm{QG}}$ defines the two-layer supercriticality criterion, which is formally similar to the supercriticality (1), apart from the discretization of the wind shear and bulk static stability and the levels at which those variable are evaluated. We evaluate $u_{500}-u_{850}, \theta_{500}-\theta_{850}, f / \beta$, and $H_{t}$ at the latitude of the Hadley circulation terminus; $\theta_{0}$ defines a reference value of potential temperature $(300 \mathrm{~K})$. Figure $\mathrm{C} 1 \mathrm{a}$ shows the meridional potential temperature contrast (C6) versus static stability (C7). Figure C1a shows that the QG supercriticality (C5) is a worse predictor of the Hadley circulation terminus than the supercriticality (1). Nevertheless, it provides a qualitative guideline for dynamical changes at the terminus latitude, similar to that found for scaling (C4) in Frierson et al. (2007) and Lu et al. (2007).

\section{REFERENCES}

Adam, O., T. Schneider, and N. Harnik, 2014: Role of changes in mean temperatures versus temperature gradients in the recent widening of the Hadley circulation. J. Climate, 27, 7450-7461, doi:10.1175/JCLI-D-14-00140.1.

Ait-Chaalal, F., and T. Schneider, 2015: Why eddy momentum fluxes are concentrated in the upper troposphere. J. Atmos. Sci., 72, 1585-1604, doi:10.1175/JAS-D-14-0243.1.

Barnes, E. A., and L. Polvani, 2013: Response of the midlatitude jets, and of their variability, to increased greenhouse gases in the CMIP5 models. J. Climate, 26, 7117-7135, doi:10.1175/ JCLI-D-12-00536.1.

Barry, L., G. C. Craig, and J. Thuburn, 2000: A GCM investigation into the nature of baroclinic adjustment. J. Atmos. Sci., 57, 1141-1155, doi:10.1175/1520-0469(2000)057<1141: AGIITN $>2.0 . \mathrm{CO} ; 2$

Booth, J. F., L. Polvani, P. A. O'Gorman, and S. Wang, 2015: Effective stability in a moist baroclinic wave. Atmos. Sci. Lett., 16, 56-62, doi:10.1002/as12.520.

Brayshaw, D. J., B. Hoskins, and M. Blackburn, 2008: The stormtrack response to idealized SST perturbations in an aquaplanet GCM. J. Atmos. Sci., 65, 2842-2860, doi:10.1175/ 2008JAS2657.1.
Butler, A. H., D. W. J. Thompson, and T. Birner, 2011: Isentropic slopes, downgradient eddy fluxes, and the extratropical atmospheric circulation response to tropical tropospheric heating. J. Atmos. Sci., 68, 2292-2305, doi:10.1175/ JAS-D-10-05025.1.

Chai, J., and G. K. Vallis, 2014: The role of criticality on the horizontal and vertical scales of extratropical eddies in a dry GCM. J. Atmos. Sci., 71, 2300-2318, doi:10.1175/ JAS-D-13-0351.1.

Dee, D. P., and Coauthors, 2011: The ERA-Interim reanalysis: Configuration and performance of the data assimilation system. Quart. J. Roy. Meteor. Soc., 137, 553-597, doi:10.1002/ qj. 828.

Edmon, H. J., Jr., B. J. Hoskins, and M. E. McIntyre, 1980: Eliassen-Palm cross sections for the troposphere. J. Atmos. Sci., 37, 2600-2616, doi:10.1175/1520-0469(1980)037<2600: EPCSFT $>2.0 . \mathrm{CO} ; 2$.

Emanuel, K. A., M. Fantini, and A. J. Thorpe, 1987: Baroclinic instability in an environment of small stability to slantwise moist convection. Part I: Two-dimensional models. J. Atmos. Sci., 44, 1559-1573, doi:10.1175/1520-0469(1987)044<1559: BIIAEO $>2.0 . \mathrm{CO} ; 2$.

Frierson, D. M. W., 2007: The dynamics of idealized convection schemes and their effect on the zonally averaged tropical circulation. J. Atmos. Sci., 64, 1959-1976, doi:10.1175/JAS3935.1.

— I. M. Held, and P. Zurita-Gotor, 2006: A gray-radiation aquaplanet moist GCM. Part I: Static stability and eddy scale. J. Atmos. Sci., 63, 2548-2566, doi:10.1175/JAS3753.1.

- J. Lu, and G. Chen, 2007: The width of the Hadley cell in simple and comprehensive general circulation models. Geophys. Res. Lett., 34, L18804, doi:10.1029/2007GL031115.

Gutowski, W. J., Jr., L. E. Branscome, and D. A. Stewart, 1992: Life cycles of moist baroclinic eddies. J. Atmos. Sci., 49, 306-319, doi:10.1175/1520-0469(1992)049<0306:LCOMBE>2.0.CO;2.

Held, I. M., 1978: The vertical scale of an unstable baroclinic wave and its importance for eddy heat flux parameterizations. J. Atmos. Sci., 35, 572-576, doi:10.1175/1520-0469(1978)035<0572: TVSOAU $>2.0 . \mathrm{CO} ; 2$.

- 1982: On the height of the tropopause and the static stability of the troposphere. J. Atmos. Sci., 39, 412-417, doi:10.1175/ 1520-0469(1982)039<0412:OTHOTT $>2.0$. CO;2.

- 2000: The general circulation of the atmosphere. Proc. Program in Geophysical Fluid Dynamics, Woods Hole, MA, Woods Hole Oceanographic Institute, $54 \mathrm{pp}$. [Available online at http:// www.whoi.edu/fileserver.do?id $=21464 \& p t=10 \& p=17332$. $]$

— in a nearly inviscid atmosphere. J. Atmos. Sci., 37, 515-533, doi:10.1175/1520-0469(1980)037<0515:NASCIA > 2.0.CO;2.

— circulation of the troposphere. Adv. Geophys., 28, 3-31, doi:10.1016/S0065-2687(08)60218-6.

$\mathrm{Hu}, \mathrm{Y}$., and Q. Fu, 2007: Observed poleward expansion of the Hadley circulation since 1979. Atmos. Chem. Phys., 7, 52295236, doi:10.5194/acp-7-5229-2007.

Jansen, M., and R. Ferrari, 2012: Macroturbulent equilibration in a thermally forced primitive equation system. J. Atmos. Sci., 69, 695-713, doi:10.1175/JAS-D-11-041.1.

Kang, S. M., and J. Lu, 2012: Expansion of the Hadley cell under global warming: Winter versus summer. J. Climate, 25, 83878393, doi:10.1175/JCLI-D-12-00323.1.

Klinger, B. A., and J. Marotzke, 2000: Meridional heat transport by the subtropical cell. J. Phys. Oceanogr., 30, 696-705, doi:10.1175/1520-0485(2000)030<0696:MHTBTS > 2.0.CO;2. 
Knippertz, P., and H. Wernli, 2010: A Lagrangian climatology of tropical moisture exports to the Northern Hemispheric extratropics. J. Climate, 23, 987-1003, doi:10.1175/ 2009JCLI3333.1.

Korty, R. L., and T. Schneider, 2007: A climatology of the tropospheric thermal stratification using saturation potential vorticity. J. Climate, 20, 5977-5991, doi:10.1175/2007JCLI1788.1. , and - 2008: Extent of Hadley circulations in dry atmospheres. Geophys. Res. Lett., 35, L23803, doi:10.1029/ 2008 GL035847.

Lapeyre, G., and I. M. Held, 2004: The role of moisture in the dynamics and energetics of turbulent baroclinic eddies. J. Atmos. Sci., 61, 1693-1710, doi:10.1175/1520-0469(2004)061<1693: TROMIT > 2.0.CO;2.

Levine, X. J., and T. Schneider, 2011: Response of the Hadley circulation to climate change in an aquaplanet GCM coupled to a simple representation of ocean heat transport. J. Atmos. Sci., 68, 769-782, doi:10.1175/2010JAS3553.1.

Lorenz, D. J., and E. T. DeWeaver, 2007: Tropopause height and zonal wind response to global warming in the IPCC scenario integrations. J. Geophys. Res., 112, D10119, doi:10.1029/ 2006JD008087.

Lu, J., G. A. Vecchi, and T. Reichler, 2007: Expansion of the Hadley cell under global warming. Geophys. Res. Lett., 34, L06805, doi:10.1029/2006GL028443.

—, G. Chen, and D. M. W. Frierson, 2008: Response of the zonal mean atmospheric circulation to El Niño versus global warming. J. Climate, 21, 5835-5851, doi:10.1175/ 2008JCLI2200.1.

Mbengue, C., and T. Schneider, 2013: Storm track shifts under climate change: What can be learned from large-scale dry dynamics. J. Climate, 26, 9923-9930, doi:10.1175/ JCLI-D-13-00404.1.

Medeiros, B., B. Stevens, and S. Bony, 2015: Using aquaplanets to understand the robust responses of comprehensive climate models to forcing. Climate Dyn., 44, 1957-1977, doi:10.1007/ s00382-014-2138-0.

Merlis, T. M., and T. Schneider, 2009: Scales of linear baroclinic instability and macroturbulence in dry atmospheres. J. Atmos. Sci., 66, 1821-1833, doi:10.1175/2008JAS2884.1.

Nguyen, H., A. Evans, C. Lucas, I. Smith, and B. Timbal, 2013: The Hadley circulation in reanalyses: Climatology, variability, and change. J. Climate, 26, 3357-3376, doi:10.1175/ JCLI-D-12-00224.1.

O'Gorman, P. A., 2011: The effective static stability experienced by eddies in a moist atmosphere. J. Atmos. Sci., 68, 75-90, doi:10.1175/2010JAS3537.1.

— range of climates simulated with an idealized GCM. J. Climate, 21, 3815-3832, doi:10.1175/2007JCLI2065.1.

Phillips, N. A., 1954: Energy transformations and meridional circulations associated with simple baroclinic waves in a twolevel, quasi-geostrophic model. Tellus, 6, 273-286, doi:10.1111/ j.2153-3490.1954.tb01123.x.

Ralph, F. M., P. J. Neiman, and G. A. Wick, 2004: Satellite and CALJET aircraft observations of atmospheric rivers over the eastern North Pacific Ocean during the winter of 1997/98. Mon. Wea. Rev., 132, 1721-1745, doi:10.1175/1520-0493(2004)132<1721: $\mathrm{SACAOO}>2.0 . \mathrm{CO} ; 2$.

Schneider, E. K., 1977: Axially symmetric steady-state models of the basic state for instability and climate studies. Part II. Nonlinear calculations. J. Atmos. Sci., 34, 280-296, doi:10.1175/1520-0469(1977)034<0280:ASSSMO>2.0.CO;2.
Schneider, T., 2004: The tropopause and the thermal stratification in the extratropics of a dry atmosphere. J. Atmos. Sci., 61, 1317-1340, doi:10.1175/1520-0469(2004)061<1317: TTATTS $>2.0 . \mathrm{CO} ; 2$.

- - 2006: The general circulation of the atmosphere. Annu. Rev. Earth Planet. Sci., 34, 655-688, doi:10.1146/ annurev.earth.34.031405.125144.

- 2007: The thermal stratification of the extratropical atmosphere. The Global Circulation of the Atmosphere, T. Schneider and A. H. Sobel, Eds., Princeton University Press, 47-77.

_ macroturbulence into critical states of weak nonlinear eddyeddy interactions. J. Atmos. Sci., 63, 1569-1586, doi:10.1175/ JAS3699.1.

_ , and P. A. O'Gorman, 2008: Moist convection and the thermal stratification of the extratropical troposphere. J. Atmos. Sci., 65, 3571-3583, doi:10.1175/2008JAS2652.1.

,$- \ldots$, and X. J. Levine, 2010: Water vapor and the dynamics of climate changes. Rev. Geophys., 48, RG3001, doi:10.1029/ 2009RG000302.

Seager, R., N. Harnik, Y. Kushnir, W. Robinson, and J. Miller, 2003: Mechanisms of hemispherically symmetric climate variability. J. Climate, 16,2960-2978, doi:10.1175/1520-0442(2003)016<2960: MOHSCV $>2.0 . \mathrm{CO} ; 2$.

Seidel, D. J., and W. J. Randel, 2007: Recent widening of the tropical belt: Evidence from tropopause observations. J. Geophys. Res., 112, D20113, doi:10.1029/ 2007JD008861.

- Q. Fu, W. J. Randel, and T. J. Reichler, 2008: Widening of the tropical belt in a changing climate. Nat. Geosci., 1, 21-24, doi:10.1038/ngeo.2007.38.

Simmons, A. J., and B. J. Hoskins, 1980: Barotropic influences on the growth and decay of nonlinear baroclinic waves. J. Atmos. Sci., 37, 1679-1684, doi:10.1175/1520-0469(1980)037<1679: BIOTGA $>2.0 . \mathrm{CO} ; 2$.

Simpson, I. R., T. A. Shaw, and R. Seager, 2014: A diagnosis of the seasonally and longitudinally varying midlatitude circulation response to global warming. J. Atmos. Sci., 71, 2489-2515, doi:10.1175/JAS-D-13-0325.1.

Smagorinsky, J., S. Manabe, and J. L. Holloway Jr., 1965: Numerical results from a nine-level general circulation model of the atmosphere. Mon. Wea. Rev., 93, 727-768, doi:10.1175/ 1520-0493(1965)093<0727:NRFANL > 2.3.CO;2.

Stone, P. H., 1978: Baroclinic adjustment. J. Atmos. Sci., 35, 561571, doi:10.1175/1520-0469(1978)035<0561:BA>2.0.CO;2.

— , and B. Nemet, 1996: Baroclinic adjustment: A comparison between theory, observations, and models. J. Atmos. Sci., 53, 1663-1674, doi:10.1175/1520-0469(1996)053<1663: BAACBT $>2.0 . \mathrm{CO} ; 2$.

Tandon, N. F., E. P. Gerber, A. H. Sobel, and L. M. Polvani, 2013: Understanding Hadley cell expansion versus contraction: Insights from simplified models and implications for recent observations. J. Climate, 26, 4304-4321, doi:10.1175/ JCLI-D-12-00598.1.

Thuburn, J., and G. C. Craig, 2000: Stratospheric influence on tropopause height: The radiative constraint. J. Atmos. Sci., 57, 17-28, doi:10.1175/1520-0469(2000)057<0017: SIOTHT $>2.0 . \mathrm{CO} ; 2$.

Trenberth, K. E., and D. P. Stepaniak, 2003: Seamless poleward atmospheric energy transports and implications for the Hadley circulation. J. Climate, 16, 3706-3722, doi:10.1175/ 1520-0442(2003)016<3706:SPAETA > 2.0.CO;2. 
Troen, I. B., and L. Mahrt, 1986: A simple model of the atmospheric boundary layer: Sensitivity to surface evaporation. Bound.-Layer Meteor., 37, 129-148, doi:10.1007/ BF00122760.

Vallis, G. K., 2006: Wave-mean flow interaction. Atmospheric and Oceanic Fluid Dynamics: Fundamentals and Large-Scale Circulation, Cambridge University Press, 295-336.

Vecchi, G. A., and B. J. Soden, 2007: Global warming and the weakening of the tropical circulation. J. Climate, 20, 43164340, doi:10.1175/JCLI4258.1.
Walker, C. C., and T. Schneider, 2006: Eddy influences on Hadley circulations: Simulations with an idealized GCM. J. Atmos. Sci., 63, 3333-3350, doi:10.1175/JAS3821.1.

Yin, J. H., 2005: A consistent poleward shift of the storm tracks in simulations of 21st century climate. Geophys. Res. Lett., 32, L18701, doi:10.1029/2005GL023684.

Zurita-Gotor, P., and R. S. Lindzen, 2007: Theories of baroclinic adjustment and eddy equilibration. The Global Circulation of the Atmosphere, T. Schneider and A. H. Sobel, Eds., Princeton University Press, 22-46. 